\title{
Relationship between habitual physical activity and variability of cardiac frequency in women with type 2 diabetes
}

\begin{abstract}
The objective of the study was to analyze the relationship between the habitual physical activity pattern and the autonomic heart rate modulation in postmenopausal women with Type 2 Diabetes. The sample of 44 postmenopausal women aged 51 to 82 years, diagnosed with Type 2 Diabetes and sedentary. The usual physical activity (AFH) was estimated by the Baecke questionnaire, which evaluates occupational physical activities (AFO), physical exercise and laser (EFL), physical activity of leisure and locomotion (ALL) and local physical activity (AFT)). Autonomic modulation was assessed by frequency variability analysis (HRV) at 20 minutes rest in the supine position. The AFO score showed a positive and significant correlation with MeanRR (ms), RMSSD (ms), HF $\left(\mathrm{ms}^{2}\right)$ and SD1 (ms) values, indicating that the increase in occupational physical activity correlates with increased vagal and Reduction in resting heart rate. The AFL score showed a positive correlation with the values of HF (nu) and negative with values of LF (nu), showing that an increase in AFL correlates with reduction of modulation The AFT score had a positive correlation with the HF (nu) value and negatively with the LF/ HF value, indicating that an increase in the standard of total habitual physical activity contributes to a Increased parasympathetic modulation and an improvement in the sympatho-vagal ratio. Although the results do not present direct correlation with the score that represents the practice of Physical Exercise systematizes with improvements of the autonomic modulation, the physical exercise contributes to a higher AFT score, and in this way may be related to the increase of the parasympathetic activity, reduction of the Sympathetic activity and improvement of the sympatho-vagal balance.
\end{abstract}

Keywords: diabetes mellitus, occupational activities, parasympathetic, physical exercise
Volume I Issue 5 - 2017

\author{
Eduardo Federighi Baisi Chagas, 1,2 \\ Aderson Fernando de Oliveira Barbosa,' \\ Milena Cristina Tsuboy,' Pedro Henrique \\ Rodrigues ${ }^{1,2}$ \\ 'Department of Physical Education, University of Marília, Brasil \\ 2Postgraduate program in Human Development and Technology, \\ Paulista State University, Brasil
}

\author{
Correspondence: Eduardo Federighi Baisi Chagas, \\ Department of Physical Education, University of Marilia, São \\ Paulo, Brasil, Tel +55 0149970031600 , \\ Email efbchagas@hotmail.com
}

Received: June 03, 2017| Published: August 29, 2017

\section{Introduction}

Among non-communicable chronic diseases, type 2 diabetes mellitus (DM2) stands out for its morbidity and mortality as a risk factor for circulatory system disorders. ${ }^{1}$ DM2 is strongly related to mobility limitations and reduced autonomy to perform daily life tasks, especially in older people ${ }^{2}$ thus contributing to a reduction in the pattern of habitual physical activity and increased physical inactivity. In women, the highest prevalence of DM2 is also related to menopause, which produces drastic changes in body composition, metabolic profile and hormonal synthesis. ${ }^{3}$ In addition, in the patient Diabetic heart rate variability (HRV) is reduced, which suggests the dysfunction of cardiac autonomic regulation, which has previously been shown to be associated with increased risk of adverse cardiac events. $^{4}$

Thus, HRV indices can be used as a complementary tool to conventional autonomic tests, and contribute to a better risk stratification of diabetic patients. ${ }^{5}$ It is known that, regardless of the presence of pathological conditions, there has been a decrease in vagal modulation, as well as an increase in sympathetic activity and the sympatho-vagal ratio with aging, and that high values of high frequency spectral power ) Represent a protection factor. ${ }^{6}$ For this reason, the indices derived from HRV analysis have been used as biomarkers of health status, disease and the effects of therapeutic interventions in several pathologies, among them, cardiovascular ${ }^{7}$ metabolic ones ${ }^{8}$ and autonomic. ${ }^{9}$ Among the therapeutic strategies for the treatment of DM2, physical exercise has received great attention, since it is believed that physical exercise modulates cardiac autonomic control, decreasing the sympathetic influence and provoking greater vagal modulation, thus affecting positively the prognosis of individuals to A variety of morbidities. ${ }^{10}$ However, sedentary lifestyle or physical inactivity goes beyond the absence of regular physical exercise, but intensifies with interruption of work life and lack of interest in leisure and occupational activities, thus influencing the pattern of habitual physical activity as a whole. ${ }^{11}$

The Habitual Physical Activity questionnaire of Baeck ${ }^{12}$ has been widely used in epidemiological and behavioral research related to health, and allows estimations of different components of physical activities ${ }^{13,14}$ such as occupational physical activities (AFO); Physical exercise and laser (EFL); Physical activities of leisure and locomotion (ALL); And local physical activity (AFT). Considering that not only systematized physical exercise but also other types of physical activity can produce an increase in energy expenditure, the hypothesis of the study concentrates on the expectation that a higher level of physical activity related to occupational, leisure and locomotion activities may bring about Health benefits of Type 2 Diabetic, and in particular the autonomic modulation of this patient. Thus, the objective of the study was to analyze the relationship between the habitual physical activity pattern and the autonomic heart rate modulation in postmenopausal women with Type 2 Diabetes. 


\section{Materials and methods}

The present study was conducted in the city of Marília, SP, Brazil, with 44 postmenopausal women (without menstruation for at least 12 months), with Type 2 Diabetes, aged between 51 and 82 years, and sedentary women $(<150$ minutes per week of physical exercise Moderate in the last 3 months). No patient presented the use of an auxiliary device for gait or presence of prosthesis. This research was approved by the Research Ethics Committee of the University of Marília-SP (UNIMAR), under protocol no. 992,073, which follows the criteria established by resolution CNS 466/12.Data on the diagnosis time of type 2 diabetes (TDM2) age, blood pressure (systolic SBP, diastolic DBP), body mass index (BMI), comorbidities and medication use were collected. The data referring to habitual physical activity were measured by the Baecke questionnaire, ${ }^{12}$ which produces physical activity scores for: Occupational physical activity (AFO); Physical and laser exercise (EFL); Physical activity of leisure and locomotion (ALL); Total physical activity (AFT).

For analysis of resting heart rate variability (HRV), 20 minutes were used lying down in the supine position. The patients did not consume drinks and foods containing alcohol or caffeine in the 12 hours prior to the experiment, nor did they perform vigorous physical exercise in the last 48 hours. Data collection took place in an air-conditioned room with a temperature between 22 and $26^{\circ} \mathrm{C}$, and in the morning. A digital validated telemetry system consisting of a transmitter positioned at the height of the xiphoid process and a monitor/receiver (Polar RS800CX, Polar Electro Oy) was recorded during the protocol., Kempele, Finland). The data was transmitted to a computer using Polar Pro Trainer software (version 5) and converted to text files. Only series with more than $95 \%$ of sinus beats were analyzed, with 256 stable points selected (Software Kubios HRV, version 2.0, University of Kopio, Finland).

In the time domain the following statistical calculations were made: mean and standard deviation of the iR-R (MeanRR and SDNN) in milliseconds (ms); Square root of the squared mean of the differences between successive normal intervals (RMSSD), expressed in ms. The last two indices are representative of the parasympathetic modulation. For the analysis in the frequency domain, the spectral power density of the most stable segment was calculated through the Fast Fourier Transform (FFT) that decomposes the signal into the following bands: high frequency (HF-0.15 to $0.4 \mathrm{~Hz}$ ) Corresponding to respiratory modulation and the vagus (parasympathetic) nerve on the heart; Low frequency (LF-0.04 to $0.15 \mathrm{~Hz}$ ) that represents sympathetic and parasympathetic modulation, but with the predominance of sympathetic; And the LF / HF ratio representing the sympatho-vagal balance. ${ }^{15}$ The LF and HF data were presented in absolute values $\left(\mathrm{ms}^{2}\right)$ and in normalized unit (nu). The normalized data will be calculated by dividing the power spectral density of a given band by total power, subtracting the very low frequency band and multiplying by $100 .{ }^{16}$

The nonlinear analysis was obtained by the Poincare plot, where the standard deviation of the points perpendicular and along the line of identity gives rise to the SD1 and SD2 indexes, respectively. The index SD1 measures the standard deviation of the distances of points to the diagonal $\mathrm{y}=\mathrm{x}$ is related to short-term variability, is influenced by respiratory sinus arrhythmia and represents the parasympathetic modulation. SD2 measures the standard deviation of distances from points to the line $y=-x+R R m$, where $R R m$ is the mean of the $i R-R$, with long-term variability and reflects the overall variability. ${ }^{15}$ For the description of the variables that represent the general characteristics of the sample, the mean and standard deviation for the quantitative variables and the relative (\%) and absolute (f) frequency distribution for the qualitative variables were used. The distribution of normality was verified by the Shapiro-wilk test. To analyze the correlation between the quantitative variables, the Pearson correlation test or the Spearman test was applied. The significance level adopted was $5 \%$ and the data were analyzed in SPSS software, version 19.0 for Windows.

\section{Results and discussion}

The mean age \pm standard deviation of the sample was $65 \pm 7.8$ years, diagnosis time for type 2 diabetes was $115 \pm 88$ months, body mass index was $30 \pm 6.7(\mathrm{~kg} / \mathrm{m} 2)$, systolic blood pressure Of $128 \pm 18$ and diastolic blood pressure of $75 \pm 10$. In the sample, $81 \%$ of the patients had a diagnosis of hypertension and $23 \%$ used beta-blocker medication (atenolol and propranolol) at doses ranging from 20 to $50 \mathrm{mg} /$ day. Regarding drug therapy for the treatment of Diabetes, $88 \%$ of the sample performed monotherapy with metformin, and $12 \%$ used combined metformin and insulin. In the correlation analysis no significant correlation was found between the indexes of heart rate variability and drug therapy. Table 1 shows the values of mean, standard deviation, minimum value and maximum value of habitual physical activity scores referring to the Baecke questionnaire.

Table I Descriptive statistics of the habitual physical activity pattern of the Baecke questionnaire

\begin{tabular}{lllll}
\hline & Minimum & Maximum & Mean & SD \\
\hline Occupational Physical Activity & 0,88 & 4,25 & 2,98 & 0,67 \\
Physical and laser exercise & 0,25 & 3,75 & 2,11 & 0,67 \\
Physical activity of leisure and locomotion & 1,00 & 3,75 & 2,54 & 0,63 \\
Total Physical Activity & 3,88 & 10,38 & 7,62 & 1,46 \\
\hline
\end{tabular}

Note: SD standard deviation

The AFO score showed a positive and significant correlation with MeanRR (ms), RMSSD (ms), HF( $\left.\mathrm{ms}^{2}\right)$ and SD1 (ms) values, indicating that the increase in occupational physical activity correlates with increased vagal and Reduction in resting heart rate (Table 2). The AFL score showed a positive correlation with the values of HF (nu) and negative with values of LF (nu), showing that an increase in AFL correlates with reduction of modulation The AFT score had a positive correlation with the $\mathrm{HF}$ (nu) value and negatively with the LF / HF value, indicating that an increase in the standard of total habitual physical activity contributes to a Increased parasympathetic modulation and an improvement in the sympatho-vagal ratio (Table 2). The EFL scores did not present significant correlations, but it is 
not possible to say that the increase in the physical exercise score does not contribute to the improvement of the autonomic modulation. ${ }^{17}$ In addition, there is evidence that aerobic and resistance exercise has a positive effect on HRV and autonomic function in high-risk populations, which positively affects the cardiovascular prognosis of these patients. ${ }^{18}$ As the study sample contains only physically inactive women, and thus the relationship between physical exercise and autonomic modulation cannot be observed.

Table 2 Correlation between habitual physical activity and heart rate variability

\begin{tabular}{lllllllll}
\hline & AFO & & EFL & & AFL & & AFT \\
\cline { 2 - 9 } & $\mathbf{R}$ & $\mathbf{P}$ & $\mathbf{r}$ & $\mathbf{P}$ & $\mathbf{r}$ & $\mathbf{P}$ & $\mathbf{r}$ & $\mathbf{P}$ \\
\hline Mean RR (ms) & 0,335 & $0,026^{*}$ & 0,013 & 0,935 & $-0,039$ & 0,800 & 0,110 & 0,479 \\
SDNN (ms) & 0,187 & 0,224 & 0,088 & 0,570 & $-0,053$ & 0,731 & 0,084 & 0,586 \\
Mean HR (ms) & $-0,329$ & $0,029^{*}$ & $-0,011$ & 0,945 & 0,046 & 0,767 & $-0,102$ & 0,509 \\
RMSSD & 0,367 & $0,014^{*}$ & 0,057 & 0,714 & 0,029 & 0,853 & 0,166 & 0,282 \\
PNN50 & 0,213 & 0,165 & 0,101 & 0,513 & 0,095 & 0,539 & 0,178 & 0,247 \\
LF (ms $\left.{ }^{2}\right)$ & 0,179 & 0,245 & 0,086 & 0,578 & $-0,077$ & 0,619 & 0,065 & 0,676 \\
LF (n.u.) & $-0,287$ & 0,059 & $-0,084$ & 0,589 & $-0,305$ & $0,044 *$ & $-0,295$ & 0,052 \\
HF (ms ${ }^{2}$ ) & 0,356 & $0,018^{*}$ & 0,086 & 0,580 & 0,078 & 0,617 & 0,195 & 0,205 \\
HF (n.u.) & 0,290 & 0,056 & 0,088 & 0,568 & 0,304 & $0,045 *$ & 0,299 & $0,048^{*}$ \\
LF/HF & $-0,290$ & 0,056 & $-0,088$ & 0,571 & $-0,269$ & 0,077 & $-0,300$ & $0,048^{*}$ \\
SDI (ms) & 0,366 & $0,015^{*}$ & 0,056 & 0,720 & 0,023 & 0,881 & 0,162 & 0,295 \\
SD2 (ms) & 0,173 & 0,262 & 0,103 & 0,506 & $-0,065$ & 0,675 & 0,075 & 0,627 \\
\hline
\end{tabular}

Note: ${ }^{p} \mathrm{p} \leq 0.05$ there is a significant correlation; $r$ correlation coefficient.AFO, occupational physical activity; EFL, Physical and laser exercise;ALL, Physical activity of leisure and locomotion;AFT, total physical activity

Despite the absence of a significant correlation between the EFL and HRV scores, it was possible to observe that an increase in the AFO, AFL and EFT score favors the improvement of the autonomic heart rate modulation, and represent an important behavioral change regarding the pattern of physical activity habitual. This should be considered because, even in young individuals a positive correlation was observed between habitual physical activity and increased duration of RR intervals (Mean RR), but the mechanism that links habitual physical activity and Mean RR duration seems to be independent of the physiological mechanisms that can be measured by heart rate variability. ${ }^{19}$ In addition Soares-Miranda et al. ${ }^{20}$ observed in elderly individuals that a greater total leisure time activity, and walking alone, were prospectively associated with more favorable and specific indexes of autonomic function in older adults, suggesting that the increase of habitual physical activity represent a potential mechanisms that may contribute to reduce cardiovascular mortality.

\section{Conclusion}

Although the results do not present direct correlation with the score that represents the practice of Physical Exercise systematizes with improvements of the autonomic modulation, the physical exercise contributes to a higher AFT score, and in this way may be related to the increase of the parasympathetic activity, reduction of the Sympathetic activity and improvement of the sympatho-vagal balance. On the other hand, the significant relationship between the increase in occupational physical activity and locomotion / leisure with the improvement of the autonomic modulation of heart rate, demonstrate the importance of considering these aspects of physical activity in the patient with Type 2 Diabetes.

\section{Acknowledgements}

None.

\section{Conflict of interest}

The author declares no conflict of interest.

\section{References}

1. Mielczarski RG, Costa JSD, Olinto MTA. Epidemiologia e organização de serviços de saúde: diabetes mellitus numa comunidade de Porto Alegre. Ciência \& Saúde Coletiva. 2012;17(1):71-78.

2. Bianchi L, Volpato S. Muscle dysfunction in type 2 diabetes: a major threat to patient's mobility and independence. Acta Diabetol. 2016;53(16):879-889.

3. Karvone GCA, Park SK, Kim C. Diabetes and Menopausa. Curr Diab Rep. 2016;16(20):1-8.

4. Tarvainen MP, Laitinen TP, Lipponen JA, et al. Cardiac autonomic dysfunction in type 2 diabetes - effect of hyperglycemia and disease duration. Front Endocrinol. 2014;5(130):1-9.

5. Silva AKF, Barbosa MPCR, Vanderlei FM, et al. Application of Heart Rate Variability in Diagnosis and Prognosis of Individuals with Diabetes Mellitus: Systematic Review. Ann Noninvasive Electrocardiol. 2016;21(3):223-235.

6. Meersman RE, Stein PK. Vagal modulation and aging. Biol Psychol. 2007;74:165-173.

7. Narayanaswamy N, Moodithaya S, Halahalli H, et al. Assessment of Risk Factor for Cardiovascular Disease Using Heart Rate Variability in Postmenopausal Women: A Comparative Study between Urban and Rural Indian Women. ISRN Cardiol. 2013;1-6. 
8. Fleischer J. Diabetic Autonomic Imbalance and Glycemic Variability. $J$ Diabetes Sci Technol. 2012;6(5):1207-1215.

9. Dimitropoulos G, Tahrani AA, Stevens MJ. Cardiac autonomic neuropathy in patients with diabetes mellitus. World J Diabetes. 2014;15(1):17-39.

10. Routledge FS, Campbell TS, Fetridge DJA, et al. Improvements in hear rate variability with exercise therapy. Can J Cardiol. 2010;26(6):303312

11. Padoin PG, Gonçalves MP, Comaru T, et al. Comparative analysis of old people practicing physical exercises and sedentary ones regarding the risk of falling. O Mundo da Saúde São Paulo. 2010;34(2):158-164.

12. Baecke JA, Burema J, Frijters JE. A short questionnaire for the measurement of habitual physical activity in epidemiological studies. Am J Clin Nutr. 1982;36(5):936-942.

13. Sadeghisani M, Manshadi DF, Azimi H, et al. Validity and Reliability of the Persian Version of Baecke Habitual Physical Activity Questionnaire in Healthy Subjects. Asian J Sports Med. 2016;7(3):e31778.

14. Vencato MM, Karageorghis CI, Priest dl, et al. Concurrent validity and cross-validation of the Brunel Life style Physical Activity Questionnaire. J Sci Med Sport. 2017;20(8):766-770.
15. TASK FORCE. Heart rate variability: standards of measurement, physiological interpretation and clinical use. Task Force of the European Society of Cardiology and the North American Society of Pacing and Electrophysiology. Circulation. 1996;93(5):1043-1065.

16. Malliani A. The Pattern of Sympathovagal Balance Explored in the Frequency Domain, News Physiol Sci. 1999;14:111-117.

17. Stuckey MI, Petrella RJ. Heart rate variability in type 2 diabetes mellitus Crit Rev Biomed Eng. 2013;41(2):137-147.

18. Voulgari C, Pagoni S, Vinik A, Poirier P. Exercise improves cardiac autonomic function in obesity and diabetes. Metabolism. 2013;62(5):609621

19. Sandercock GR, Hardy SD, Nunan D, et al. The relationshipsbetween self-assessed habitual physical activity and non-invasive measures of cardiac autonomic modulation in young healthy volunteers. $J$ Sports Sci. 2008;26(11):1171-1177.

20. SoaresML, Sattelmair J, Chaves P, et al. Physical Activity and Hear Rate Variability in Older Adults: The Cardiovascular Health Study. Circulation. 2014;129(21):2100-2110. 